\title{
Swimming attenuates inflammation, oxidative stress, and apoptosis in a rat model of dextran sulfate sodium-induced chronic colitis
}

\author{
Ling Qin ${ }^{1, *}$, Zhi-qiang Yao ${ }^{2, *}$, Qi Chang ${ }^{3, *}$, Ya-li Zhao ${ }^{2, *}$, Ning-ning Liu' ${ }^{2, *}$, Xiao-shan \\ Zhu ${ }^{2}$, Qin-qin Liu ${ }^{2}$, Li-feng Wang ${ }^{4}$, An-gang Yang ${ }^{5}$, Chun-fang Gao ${ }^{2}$ and Jun-tang \\ $\mathbf{L i}^{2,3,4,5}$ \\ ${ }^{1}$ Department of Hematology, First Affiliated Hospital, and College of Clinical Medicine of Henan University of Science and \\ Technology, Luoyang, Henan, China \\ ${ }^{2}$ Centre of Inflammation and Cancer Research, 150th Central Hospital of PLA, Luoyang, Henan, China \\ ${ }^{3}$ Centre of Biomaterial and Biophysics Research, Institute of Training Medicine, 150th Central Hospital of PLA, Luoyang, \\ Henan, China \\ ${ }^{4}$ State Key Laboratory of Cancer Biology, Department of Biochemistry and Molecular Biology, Fourth Military Medical \\ University, Xi'an, Shaanxi, China \\ ${ }^{5}$ State Key Laboratory of Cancer Biology, Department of Immunology, Fourth Military Medical University, Xi'an, Shaanxi, \\ China \\ * These authors have contributed equally to this work \\ Correspondence to: Jun-tang Li, email: juntangli@163.com
}

Chun-fang Gao, email: gaocfsoul@163.com

Ling Qin, email: lingqinvvip@163.com

Keywords: chronic colitis, swimming, inflammation, oxidative stress, apoptosis, Immunology and Microbiology Section, Immune response, Immunity

Received: March 28, 2016

Accepted: December 15, 2016

Published: December 21, 2016

\section{ABSTRACT}

Increasing evidence suggests that regular physical exercise suppresses chronic inflammation. However, the potential inhibitory effects of swimming on dextran sulfate sodium (DSS)-induced chronic colitis, and its underlying mechanisms, remain unclear. In this study, rats were orally administered DSS to induce chronic colitis, and subsequently treated with or without swimming exercise. A 7-week swimming program ( 1 or 1.5 hours per day, 5 days per week) ameliorated DSS-caused colon shortening, colon barrier disruption, spleen enlargement, serum LDH release, and reduction of body weight gain. Swimming for 1.5 hours per day afforded greater protection than 1 hour per day. Swimming ameliorated DSS-induced decrease in crypt depth, and increases in myeloperoxidase activity, infiltration of Ly6G+ neutrophils and TNF-a- and IFN- $\gamma$-expressing $\mathrm{CD}^{+}{ }^{+} \mathrm{T}$ cells, as well as fecal calprotectin and lactoferrin. Swimming inhibited pro-inflammatory cytokine and chemokine production and decreased the protein expression of phosphorylated nuclear factor-kB p65 and cyclooxygenase 2, whereas it elevated interleukin-10 levels. Swimming impeded the generation of reactive oxygen species, malondialdehyde, and nitric oxide; however, it boosted glutathione levels, total antioxidant capacity, and superoxide dismutase and glutathione peroxidase activities. Additionally, swimming decreased caspase-3 activity and expression of apoptosis-inducing factor, cytochrome c, Bax, and cleavedcaspase-3, but increased Bcl-2 levels. Overall, these results suggest that swimming exerts beneficial effects on DSS-induced chronic colitis by modulating inflammation, oxidative stress, and apoptosis. 


\section{INTRODUCTION}

Chronic inflammation plays a major role in the pathology of various diseases. Inflammatory bowel disease (IBD), including Crohn's disease and ulcerative colitis (UC), is characterized by an inappropriate and continuous active inflammatory response accompanied by tissue destruction [1]. Conventional IBD treatments can reduce active disease periods and help to maintain remission, but these treatments often cause side effects, show marginal results, and induce resistance to therapy. Therefore, alternative treatment strategies are urgently needed.

Dextran sulfate sodium (DSS) is a non-genotoxic sulfated polysaccharide that is frequently used to induce experimental chronic colitis, which is histopathologically similar to human UC [2]. Intestinal epithelial barrier disruption is regarded as the main event during progression of IBD; this is followed by activation of the immune response [3]. Activated immune responses in the intestine result in the excessive production of proinflammatory cytokines, such as tumor necrosis factor- $\alpha$ (TNF- $\alpha$ ), interleukin (IL)-1 $\beta$, IL-6, IL- 8 , and interferon- $\gamma$ (IFN- $\gamma$ ). These cytokines amplify inflammatory cascades by triggering the generation of other pro-inflammatory cytokines and chemokines, thereby recruiting neutrophils to the site of mucosal injury. Activated neutrophils produce fecal biomarkers of IBD such as calprotectin and lactoferrin [4], and generate large amounts of reactive oxygen species (ROS), nitric oxide (NO), and prostaglandin $\mathrm{E}_{2}\left(\mathrm{PGE}_{2}\right)$, which ultimately cause mucosal disruption [3]. Oxidative stress plays a prominent role in the pathogenesis of chronic colitis [5]. Excessive ROS activate nuclear factor- $\mathrm{\kappa B}(\mathrm{NF}-\mathrm{\kappa B})$, which in turn induces the production of numerous inflammatory mediators as well as the expression of inducible nitric oxide synthase (iNOS) and cyclooxygenase 2 (COX2) [6]. The pathogenesis of IBD also involves increased apoptosis with consequent loss of intestinal epithelial cells [7].

Regular physical activity improves overall wellbeing and may be used as an adjunct anti-inflammatory therapy for chronic inflammatory diseases in both humans and animals [8]. Cook et al. [9] established a mouse colitis model to show that forced treadmill exercise exacerbates inflammation, whereas voluntary wheel training is protective. Previous studies indicate that voluntary wheel exercise inhibits apoptosis of splenic lymphocyte subsets in mice by modulating the immune system and increasing antioxidant capacity [10]. In particular, swimming has been prescribed as a non-pharmacological treatment for arterial hypertension, obesity, and coronary heart disease as it reduces the levels of inflammatory cytokines without causing oxidative stress [11]. Also, swimming reduces the risk of dimethylhydrazine-induced colon cancer in rats by inhibiting inflammation [12]. All of these findings encouraged us to examine the potential protective effects of swimming exercise, and the underlying mechanisms, in rats with DSS-induced colitis, an experimental model of human IBD.

In this study, we found that swimming attenuated DSS-induced colon shortening, decrease of crypt depth, colonic barrier disruption, spleen swelling, LDH release in blood, decrease in body weight (BW) gain, histopathological deteriation, the increases in fecal biomarkers of IBD, and the immigration of $\mathrm{Ly}^{6} \mathrm{G}^{+}$ neutrophils and TNF- $\alpha$ - and IFN- $\gamma$-expressing $\mathrm{CD}^{+}$ $\mathrm{T}$ cells into mesenteric lymph nodes (MLNs) and the lamina propria (LP). Examination of the underlying molecular mechanisms revealed that swimming reduced the expression of phosphorylated NF- $\mathrm{KB}$ p 65 and $\mathrm{COX} 2$ in the DSS-treated rat colon, as well as the levels of TNF- $\alpha$, IL-1 $\beta$, IL-6, IL-10, KC, CCL2, and PGE . Moreover, swimming decreased the production of ROS, malondialdehyde (MDA), and NO, and inhibited iNOS activity, while increased glutathione (GSH) and total antioxidant capacity (TAC) levels, and the activities of superoxide dismutase (SOD) and glutathione peroxidase (GPx). Swimming also impeded DSS-induced apoptosis in the colon by inhibiting caspase-3 activity, reducing the expression of apoptosis-inducing factor (AIF), cytochrome c, Bax, and cleaved-caspase-3 (Cl-caspase-3), and increasing Bcl-2 level. These results indicate that swimming exerts anti-inflammatory, antioxidant, and anti-apoptotic effects, thereby ameliorating DSS-caused chronic colitis.

\section{RESULTS}

\section{Swimming attenuates DSS-induced spleen enlargement, reduction of $\mathrm{BW}$ gain, and colon injury}

We initially found that oral administration of DSS caused significant decrease in BW gain (Figure 1A), spleen swelling (Figures 1B and 1C), elevated serum LDH (Figure 1D), colon shortening (Figure 1E), and colonic barrier disruption (Figure 1F) in rats when compared with control (Ctrl) animals. Swimming for 1 hour per day (h/d) or $1.5 \mathrm{~h} / \mathrm{d}$ led to a significant inhibition of the above vicious effects in DSS-insulted rats (Figures 1A-1G); however, swimming for $1.5 \mathrm{~h} / \mathrm{d}$ had a greater protective effect. Thus, a period of $1.5 \mathrm{~h} / \mathrm{d}$ was selected for further studies. As shown in Figures 2A and 2B, the DSS + Sedentary (Sed) group showed severe dysplastic lesions in colon tissues and a marked increase in the inflammation score when compared with the Ctrl groups; however, swimming reduced inflammation-led flat dysplasia and improved the colonic architecture in DSS-challenged rats. Moreover, DSS-treated rats exhibited decreased crypt depth, which was reversed by swimming (Figure 2C). These results indicate that swimming ameliorates the 
decreased BW gain, spleen enlargement and colon damage in DSS-administrated rats.

\section{Swimming reduces the infiltration of activated neutrophils and $\mathrm{CD3}^{+} \mathrm{T}$ cells into colon in DSS- induced colitis}

Neutrophils play an important role in DSSinduced colonic mucosal injury [13]. As shown in Figures $3 \mathrm{~A}$ and 3B, DSS caused a marked enhancement in myeloperoxidase (MPO) activity in colon tissues, and in the percentage of $\mathrm{Ly}_{6} \mathrm{G}^{+}$neutrophils in the MLNs and LPs, both of which were significantly attenuated by swimming. Fecal calprotectin and lactoferrin, considered as biomarkers of IBD, increases significantly as infiltration of neutrophils in intestinal tracts [14]. Here, DSS administration increased fecal calprotectin and lactoferrin compared to Ctrl groups; however, swimming effectively hindered the elevation (Figures 3C and 3D). Next, we investigated the effect of swimming on the recruitment of activated $\mathrm{CD}^{+} \mathrm{T}$ cells into MLNs and LPs after DSS challenge. Flow cytometry analysis revealed that the numbers of TNF- $\alpha$ - and IFN- $\gamma$-expressing $\mathrm{CD}^{+} \mathrm{T}$ cells increased after DSS treatment; these increases were significantly reduced by swimming (Figures $3 \mathrm{E}$ and $3 \mathrm{~F}$ ). These results suggest that swimming inhibits neutrophil and $\mathrm{CD}^{+} \mathrm{T}$ cell infiltration into colon in DSS-induced colitis.

Swimming modulates the production of inflammatory mediators in DSS-induced colitis

To gain insight into the inflammatory milieu associated with DSS-induced colitis, we performed enzyme-linked immunosorbent assays (ELISAs) to measure the levels of various inflammatory mediators. DSS induced severe inflammatory responses, as indicated by markedly higher levels of TNF- $\alpha$ (Figure 4A), IL-1 $\beta$ (Figure 4B), IL-6 (Figure 4C), KC (Figure 4D), CCL2 (Figure 4E), and $\mathrm{PGE}_{2}$ (Figure 4F) in the colon of rats
A

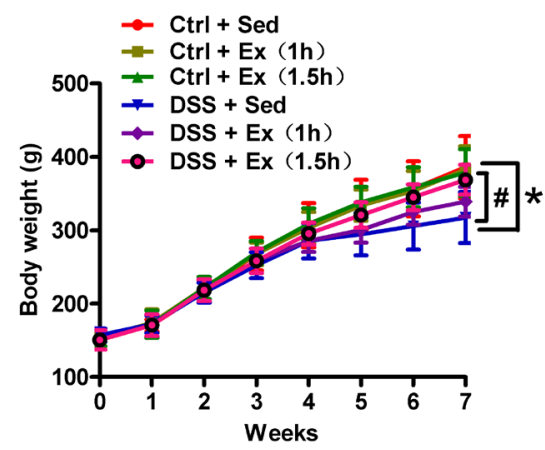

$\mathrm{B}$

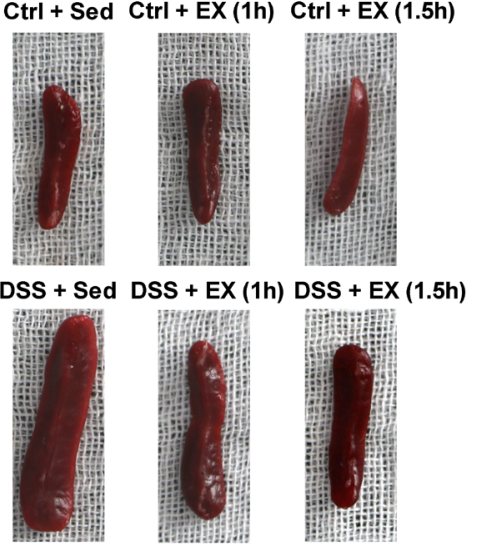

$\mathrm{F}$
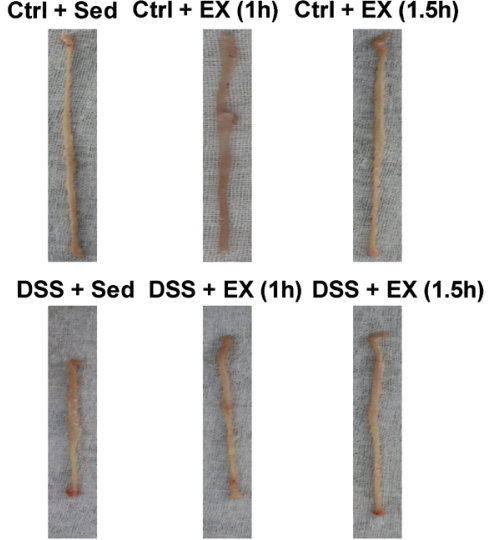

C

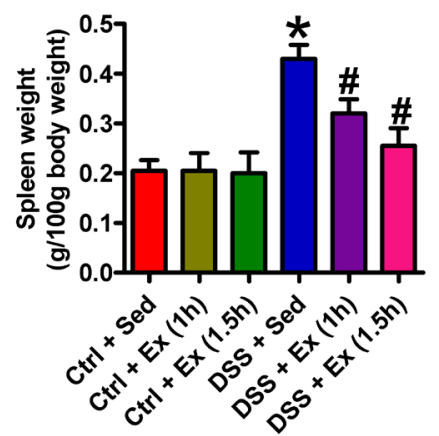

E

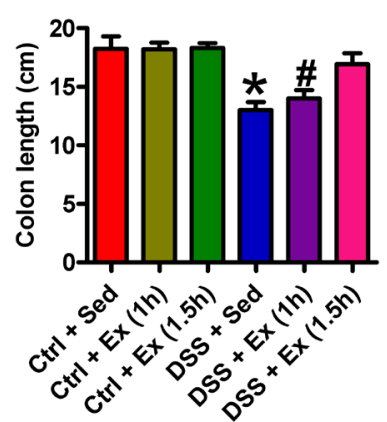

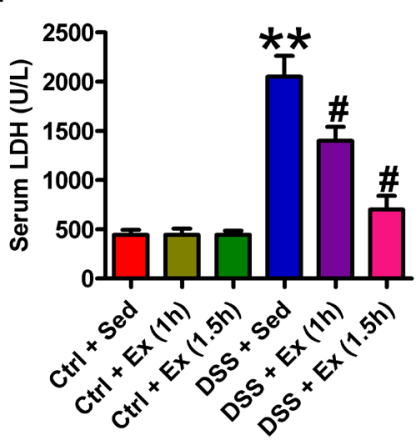

G

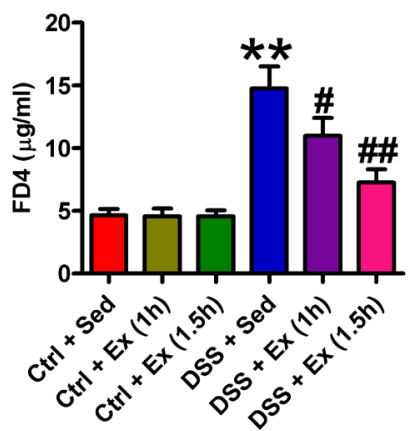

Figure 1: Effects of swimming on the severity of DSS-induced colitis in rats. A. Changes in body weight. Body weight was measured both before and after adaptation, and once per week throughout the experimental period. B.-F. After 7 weeks of swimming exercise, rats were fasted for $4 \mathrm{~h}$ and then euthanized with sodium pentobarbital. The spleen and entire colon were removed, and blood was collected. Representative photo of spleens B., changes in spleen weight C., representative macroscopic view of the colons D., quantification of colon length E., and measurement of serum LDH levels F.. G. Changes in colon barrier function. After $4 \mathrm{~h}$ of fasting, rats were administered FD4 by gavage. Plasma was sampled $5 \mathrm{~h}$ after the procedure, and its fluorescence was measured. Data are representative of three independent experiments and are expressed as the mean $\pm \mathrm{SD} .{ }^{*} P<0.05$ and ${ }^{* *} P<0.01$ vs. Ctrl groups; ${ }^{\#} P<0.05$ and ${ }^{\# \#} P<0.01$ vs. DSS + Sed group. 
A

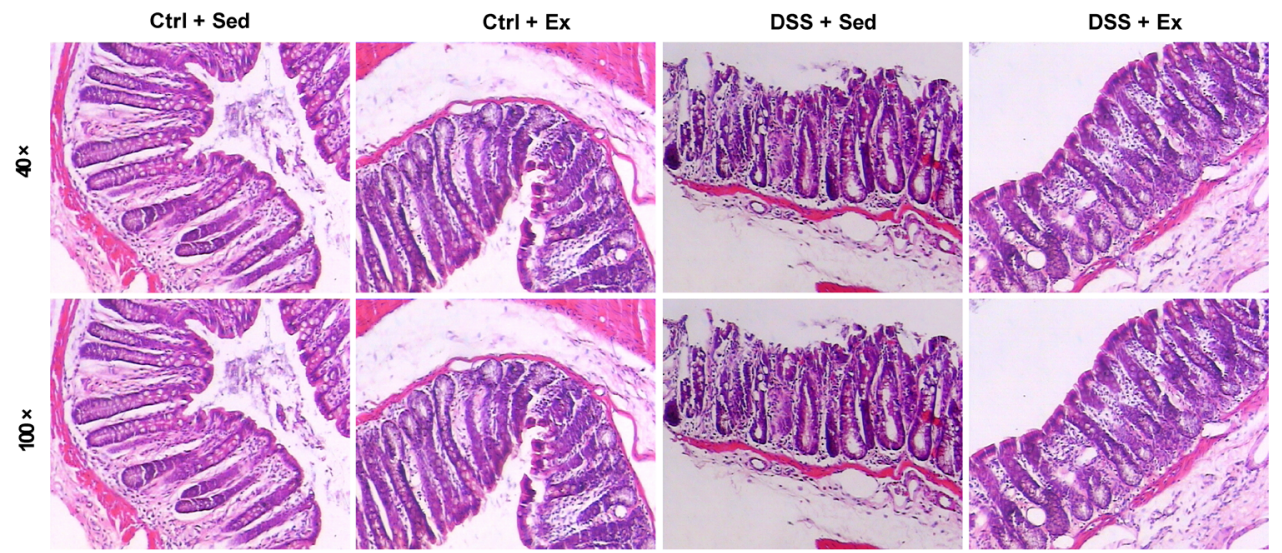

B

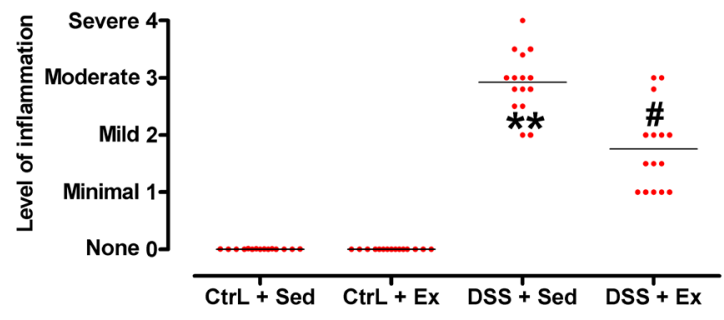

C

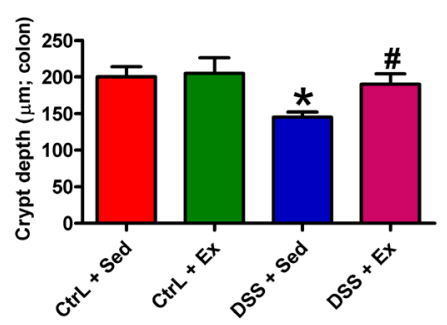

Figure 2: Swimming attenuates DSS-induced histopathological damage in the rat colon. After 7 weeks of swimming exercise, colon sections were collected and utilized for histopathological investigation. A. Representative images of HE-stained colon tissues (40× magnification, upper panels; 100× magnification, lower panels). B. Inflammation scores. Results are presented as a dot plot, with the lines representing the mean inflammation score. Each dot represents an individual rat. C. Crypt depth in colon mucosa sections. Data are representative of three independent experiments and are expressed as the mean $\pm \mathrm{SD}$. ${ }^{*} P<0.05$ and ${ }^{* *} P<0.01$ vs. Ctrl groups; ${ }^{*} P$ $<0.05 v s$.DSS + Sed group.

A

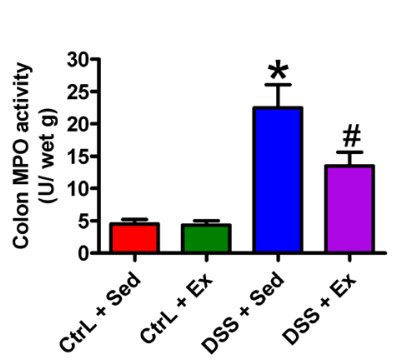

D

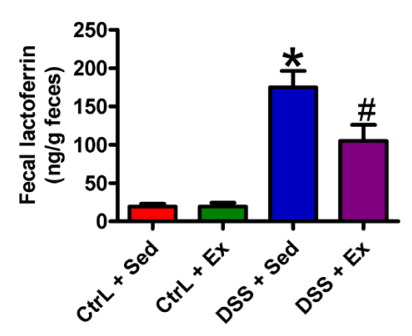

B

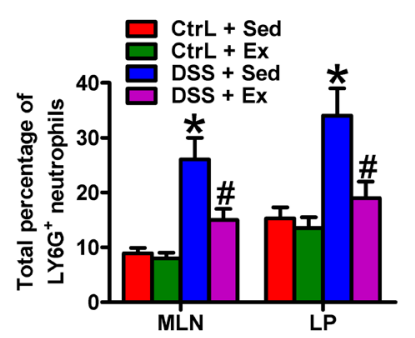

$E$

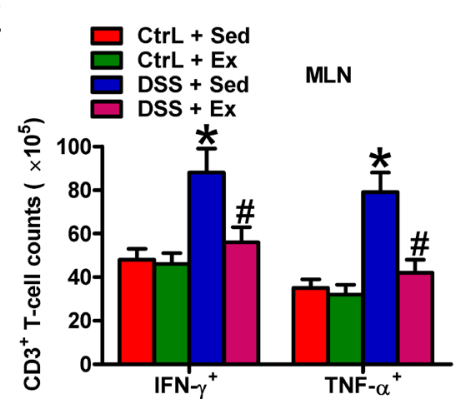

C

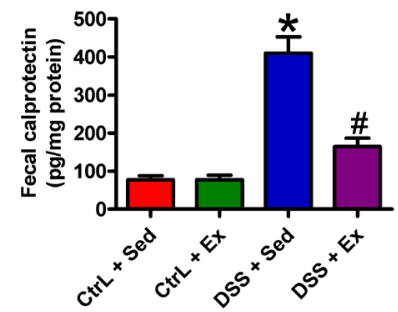

$\mathrm{F}$

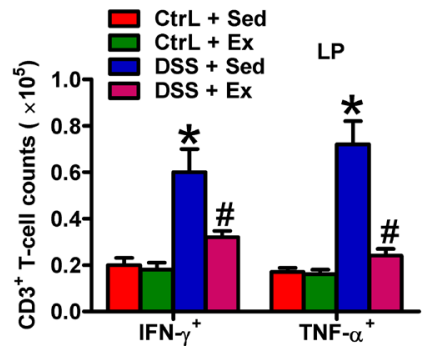

Figure 3: Swimming reduces MPO activity, infiltration of $\mathrm{Ly}^{6} \mathrm{G}^{+}$neutrophil and TNF- $\alpha$ - and IFN- $\gamma$-expressing $\mathrm{CD3}^{+}$ T cells and fecal biomarkers in DSS-induced colitis in rats. A. MPO activity in colon tissues from the different treatment groups. B. Total percentage of Ly6G+ neutrophils in the MLNs and LPs. C. and D. Measurement of fecal biomarkers such as calprotectin C. and lactoferrrin D.. Feces were harvested and ELISAs were performed to determine the levels of calprotectin and lactoferrrin. E. and F. Changes in the number of TNF- $\alpha$ - and IFN- $\gamma$-expressing $\mathrm{CD}^{+}$T cells in the MLNs E. and LP F.. Lymphocytes were freshly isolated from the MLNs and $\mathrm{LP}$ and stained to quantify $\mathrm{CD}^{+} \mathrm{T}$ cells and neutrophils. MLN- or LP-derived $\mathrm{CD}^{-}$lymphocytes were examined for Ly6G expression by flow cytometry. Data are representative of three independent experiments and are expressed as the mean $\pm \mathrm{SD}$. ${ }^{*} P<0.05 v s$. Ctrl groups; ${ }^{\sharp} P<0.05 v$ s. DSS + Sed group. 
than in that of Ctrl rats; these increases were significantly attenuated by swimming. DSS treatment increased IL10 production, however, which was further enhanced by swimming (Figure 4G). Moreover, DSS challenge increased rat serum levels of TNF- $\alpha$ (Figure 4H), IL$1 \beta$ (Figure 4I), IL-6 (Figure 4J), KC (Figure 4K), and $\mathrm{PGE}_{2}$ (Figure $4 \mathrm{~L}$ ), which were significantly inhibited by swimming. These results demonstrate that swimming exerts anti-inflammatory effects by inhibiting the production of several pro-inflammatory mediators and increasing IL-10 levels.

\section{Swimming abrogates expression of NF- $\mathrm{KB}$ p65 and COX2}

$\mathrm{NF}-\kappa \mathrm{B}$ and COX2 play crucial roles in the pathogenesis of IBD [6]. Immunohistochemistry (IHC) staining of colon tissue from rats with DSS-induced colitis revealed extensive $\mathrm{NF}-\kappa \mathrm{B}$ p65 and COX2 expression
(Figures 5A and 5B). Western blot analysis confirmed that DSS induced a significant increase in the expression of phosphorylated (p)-NF- $\mathrm{B}$ p65 and COX2 (Figures 5C and 5D). Notably, swimming led to a significant reduction in the levels of NF- $\kappa \mathrm{B}$ p 65 and COX2 (Figures 5A-5D). These results indicate that downregulation of $\mathrm{NF}-\kappa \mathrm{B}$ p65 and COX2 may underlie the beneficial effects of swimming in rats with DSS-induced colitis.

\section{Swimming inhibits oxidative stress and increases antioxidant products}

During the development of IBD, the inflammatory process induces oxidative stress and reduces cellular antioxidant capacity [6]. DSS caused marked oxidative stress, as indicated by increases in ROS (Figure 6A), MDA (Figure 6B), and NO (Figure 6C) levels, and iNOS activity (Figure 6D). This was accompanied by decreases in GSH (Figure 6E) and TAC (Figure 6F) contents, and SOD
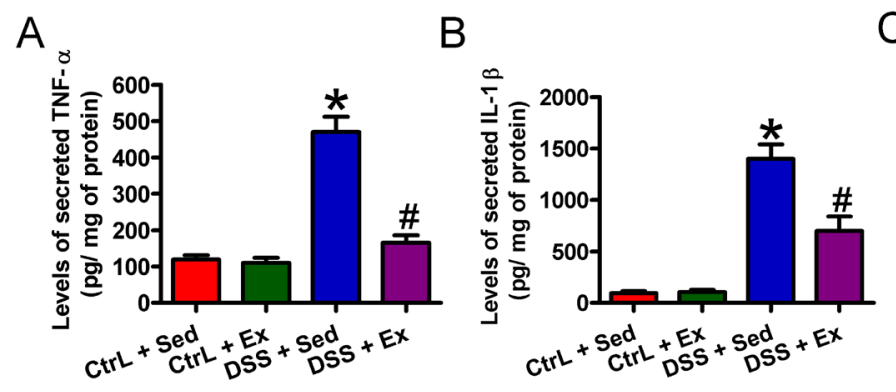

C

$\mathrm{E}$

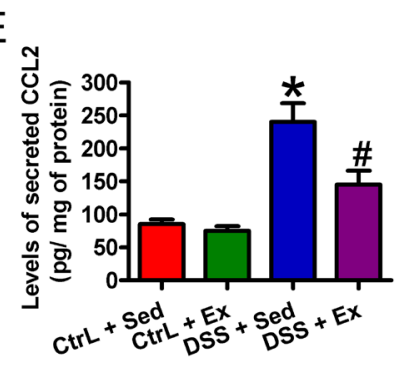

F
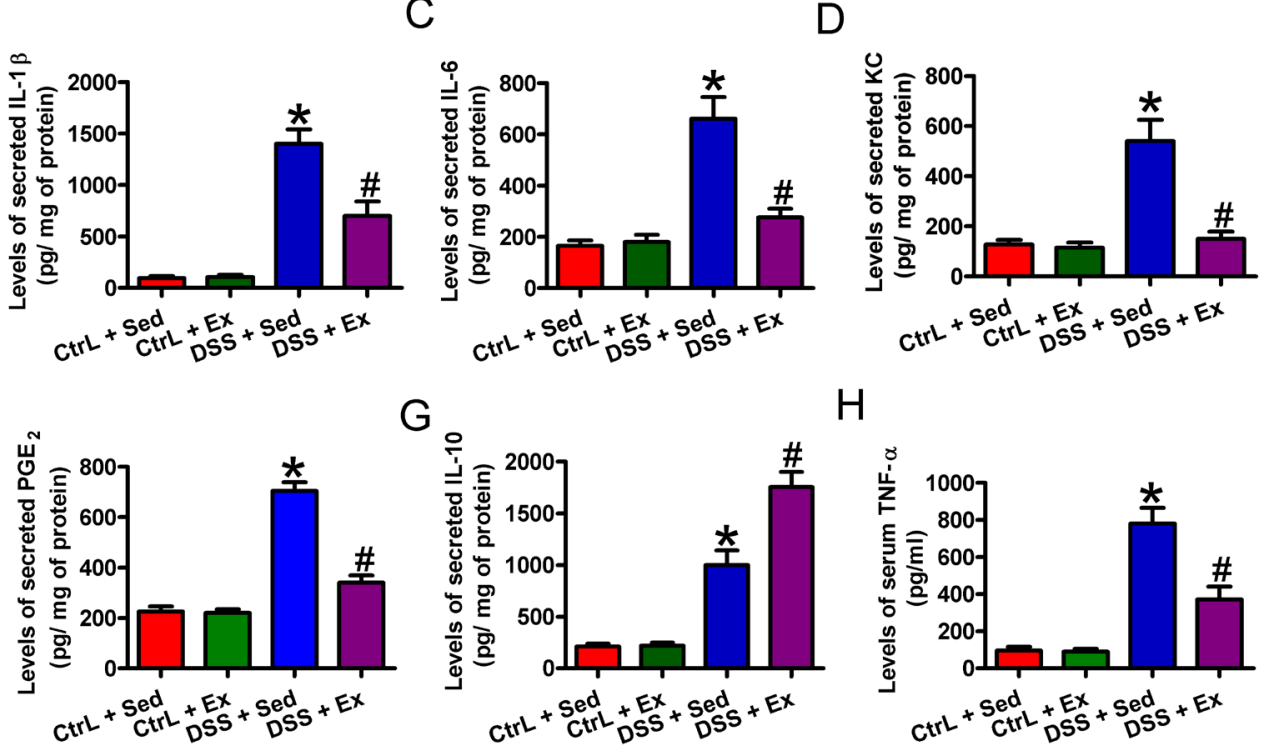

G

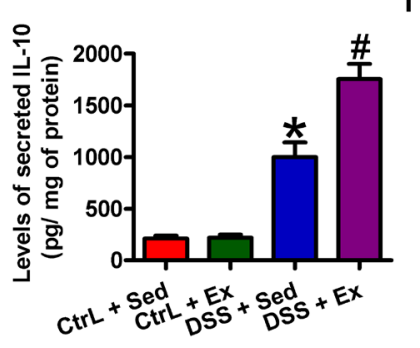

$\mathrm{H}$
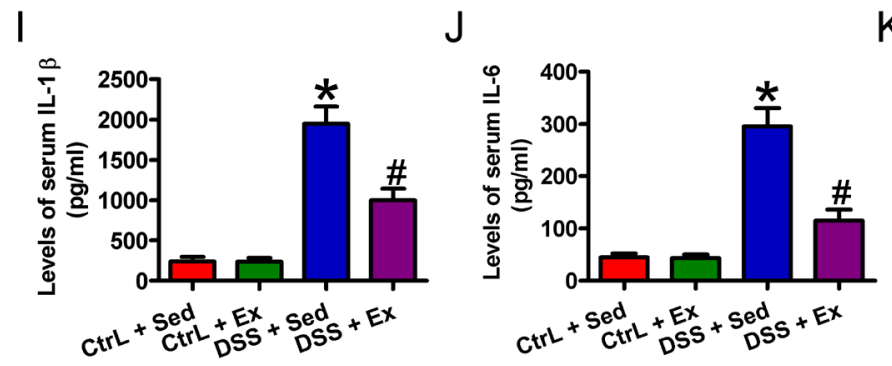

$\mathrm{K}$
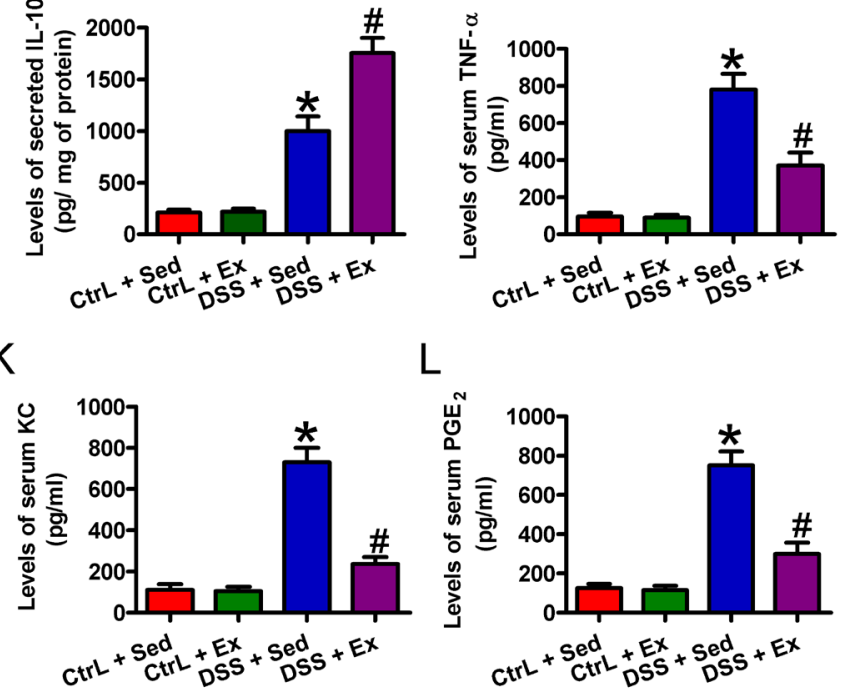

Figure 4: Swimming inhibits the production of inflammatory mediators in rats with DSS-induced colitis. After 7 weeks of swimming exercise, the colon was removed from the distal end of the cecum to the rectum, longitudinally cut, thoroughly rinsed in sterile $20 \mathrm{mM}$ PBS (pH 7.4), spread onto the bottoms of six-well dishes, and incubated overnight in $1 \mathrm{~mL}$ of culture medium. A.-G. ELISAs were performed to detect the supernatant levels of TNF- $\alpha$ A., IL-1 $\beta$ B., IL-6 C., KC D., CCL2 E., PGE 2 F., and IL-10 G.. After 7 weeks of swimming exercise, serum was collected from every group. H.-L. ELISAs were carried out to detect the serum levels of TNF- $\alpha$ H., IL- $1 \beta$ I., IL-6 J., KC K., PGE 2 L.. Data are representative of three independent experiments and are expressed as the mean \pm SD. ${ }^{*} P<0.05 v s$. Ctrl groups; ${ }^{\sharp} P<0.05$ vs. DSS + Sed group. 
A
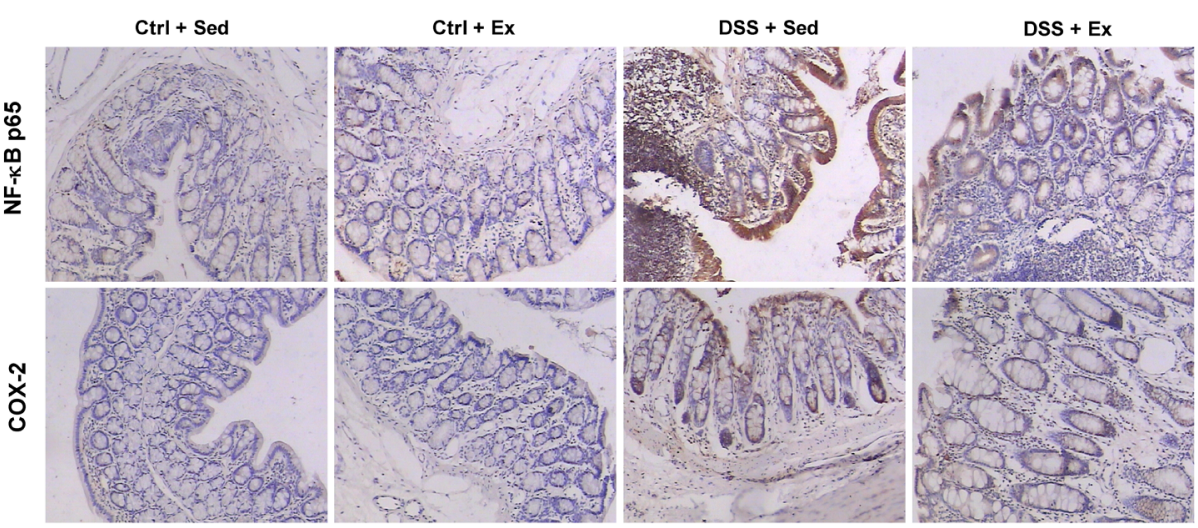

B

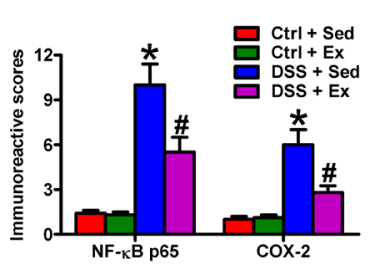

C

D
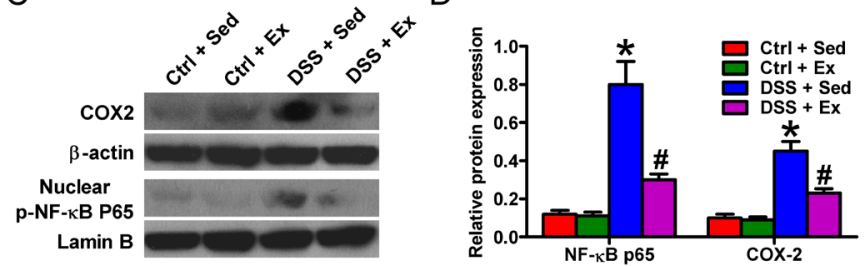

Figure 5: Swimming decreases the expression of NF-кB p65 and COX2 protein in the colon of rats with DSS-induced colitis. After 7 weeks of swimming exercise, colon tissues were collected and used for immunohistochemical investigation, or were homogenized in tissue lysis buffer prior to Western blot analysis. A. Representative images of IHC-stained tissues showing NF- $\mathrm{B}$ p65

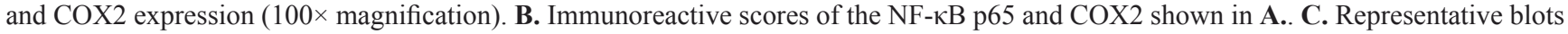
showing COX2 and p-NF- $\mathrm{B}$ p65 expressions after Western blot analysis. $\beta$-actin and lamin B were used as endogenous loading controls. D. The COX2/ $\beta$-actin and $\mathrm{p}-\mathrm{NF}-\mathrm{\kappa B}$ p65/lamin B ratios. Data are representative data of three independent experiments and are expressed as the mean \pm SD. ${ }^{*} P<0.05$ vs. Ctrl groups; ${ }^{\sharp} P<0.05$ vs. DSS + Sed group.
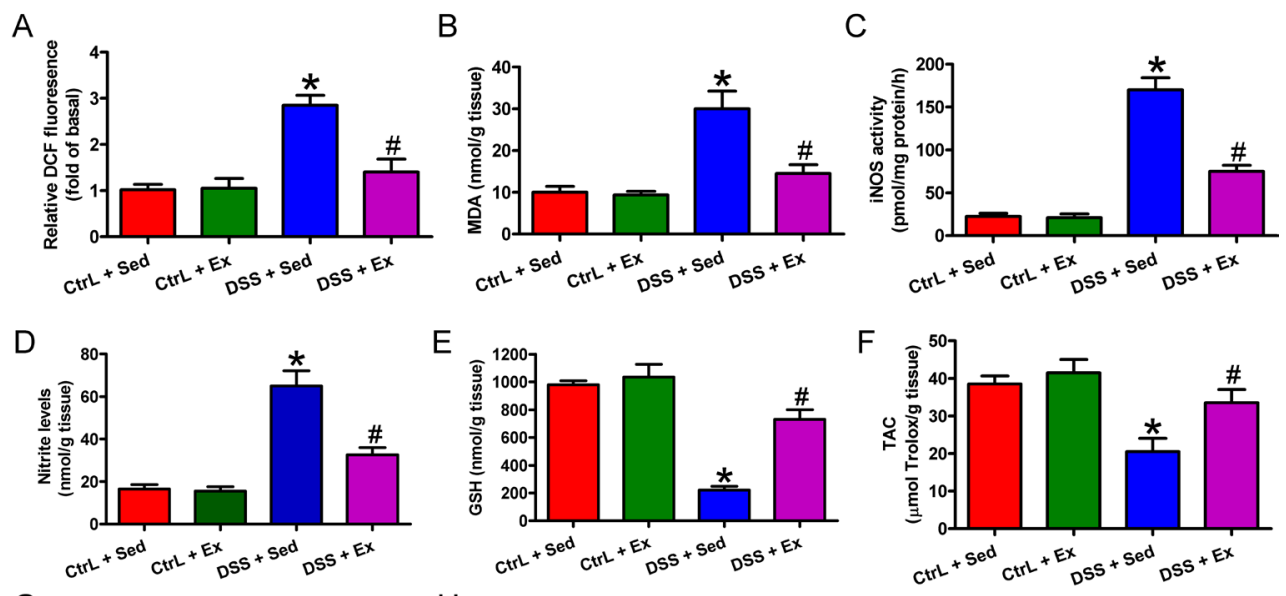

$\mathrm{G}$

$E$
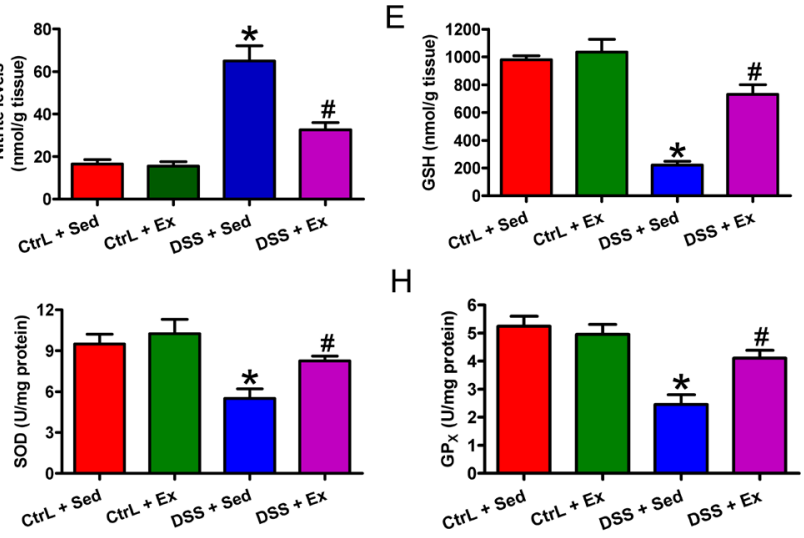

$\mathrm{H}$

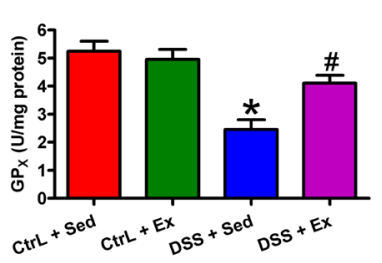

Figure 6: Swimming ameliorates oxidative stress and increases antioxidant defense in the colon of rats with DSS-induced colitis. After 7 weeks of swimming exercise, colon sections were collected and homogenized in tissue lysis buffer and centrifuged, and the supernatants were harvested. A. ROS production was measured in terms of relative DCF fluorescence. B. Lipid peroxide was measured in terms of MDA production. C. iNOS activity was measured in a standard assay that monitors the conversion of arginine to citrulline. D. Nitrite production was assessed in a colorimetric reaction with the Griess reagent. E. GSH levels were determined using DTNB reagent. F. TAC was determined using a commercial antioxidant assay kit. G. SOD activity was assessed according to inhibition of pyrogallol autooxidation. H. GPx activity was determined according to its ability to oxidize GSH. Data are representative of three independent experiments and are expressed as the mean $\pm \mathrm{SD} .{ }^{*} P<0.05$ vs. Ctrl groups; ${ }^{*} P<0.05$ vs. DSS + Sed group. 
(Figure 6G) and GPx (Figure 6H) activities. Swimming provided a significant protection against oxidative stress, as evidenced by the decreased levels of ROS, MDA, and NO, and reduced iNOS activity (Figures 6A-6D), in addition to the rescuing of GSH and TAC levels and of SOD and GPx activities (Figures 6E-6H). Thus, swimming attenuates oxidative stress and boosts antioxidant defenses to protect against DSS-induced colitis.

\section{Swimming inhibits apoptosis in rats with DSS- induced colitis}

Exposure of the colonic mucosa to intracellular stressors such as ROS in an inflammatory environment triggers apoptosis of colon epithelial cells, leading to progression of IBD [15]. Thus, we investigated the effects of swimming on apoptosis of colon epithelial cell in rats with DSS-induced colitis. We found that DSS induced apoptosis of inflamed colon epithelial cells, as indicated by increases in caspase-3 activity (Figure 7A) and expression of Cl-caspase-3, AIF, cytochrome c, and Bax, along with downregulation of Bcl-2 (Figures 7B and 7C). Swimming counteracted the above changes, thereby promoting cell survival (Figures 7A-7C). These findings suggest that swimming protects the colon mucosa from apoptosis in DSS-induced colitis.

\section{DISCUSSION}

In this study, we demonstrate that swimming alleviates DSS-induced colitis in rats. The beneficial effects are as follows (Figure 7D): (1) Attenuation of DSS-induced reduced BW gain, spleen swelling, serum LDH release, colon shortening, decreased crypt depth, and colonic barrier disruption; (2) reduction of neutrophil and TNF- $\alpha$ - and IFN- $\gamma$-expressing $\mathrm{CD}^{+} \mathrm{T}$ cell infiltration into MLNs and LPs and fecal calprotectin and lactoferrin; (3) inhibition of inflammatory mediator production and $\mathrm{NF}-\kappa \mathrm{B}$ p65 and COX2 protein expression; (4) decrease in oxidative stress and increase in antioxidant defense; and (5) downregulation of pro-apoptotic molecules and upregulation of Bcl-2 expression.

IBD is associated with an influx of neutrophils into the mucosa and, subsequently, into the intestinal lumen, resulting in crypt abscess formation [16]. Monoclonal antibody-mediated depletion of neutrophils reduces several parameters associated with DSS-induced colitis in rats [13]. Morohoshi et al. [17] demonstrated significant increases in neutrophil elastase activity in both plasma and colonic mucosal tissues in UC patients, and showed that ONO-5046 (a specific inhibitor of neutrophil elastase) has therapeutic effects against DSS-induced colitis by reversing weight loss and spleen enlargement
A

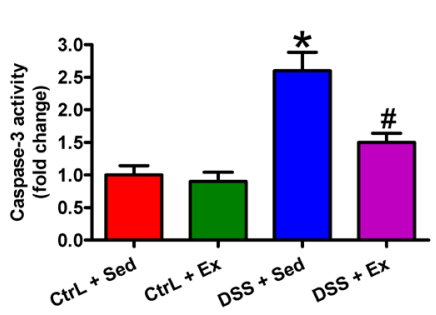

B

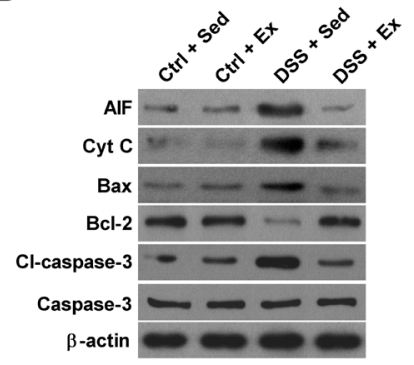

C

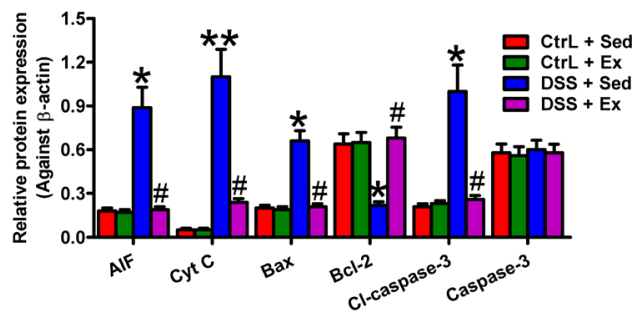

D

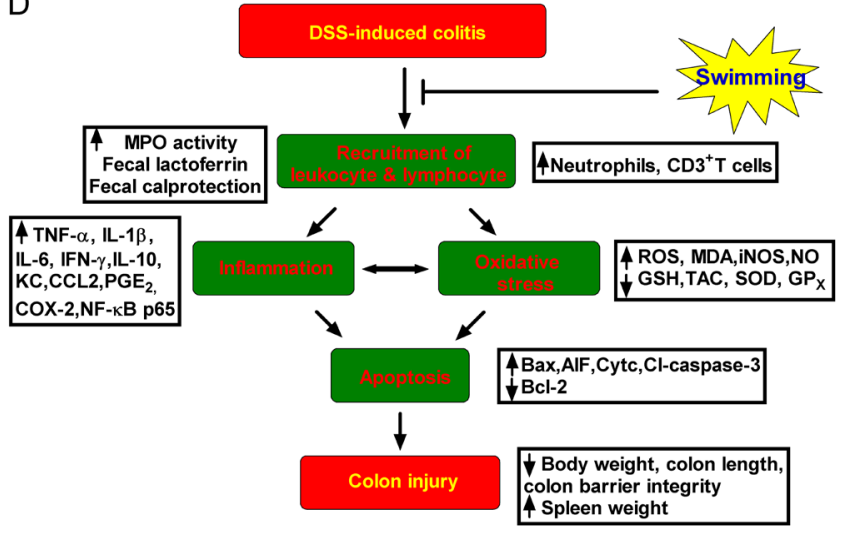

Figure 7: Swimming impedes apoptosis in the colon of rats with DSS-induced colitis. After 7 weeks of swimming exercise, colon sections were collected, homogenized in tissue lysis buffer, and centrifuged, and the supernatants were harvested. A. Caspase-3 activity was measured using a Caspase-3/CPP32 colorimetry kit. B. Expression of AIF, cytochrome c, Bax, Bcl-2, Cl-caspase-3, and caspase-3 proteins was assessed by Western blotting. $\beta$-actin was used as an endogenous loading control. $\mathbf{C}$. The relative expression of the proteins in B. was normalized against $\beta$-actin. D. Diagram depicting the inhibitory effects of swimming exercise on DSS-induced colitis in rats. Data are representative data of three independent experiments and are expressed as the mean \pm SD. ${ }^{*} P<0.05$ and ${ }^{* *} P<0.01 v s$. Ctrl groups; ${ }^{\sharp} P<0.05$ vs. DSS + Sed group. 
and reducing inflammation scores. Here, we found that MPO activity, an indicator of neutrophil infiltration, was significantly higher in rats with DSS-induced colitis than in controls; these increases were attenuated by swimming. These observations were confirmed by experiments demonstrating a reduction in the percentage of $\mathrm{Ly} 6 \mathrm{G}^{+}$ neutrophils in the MLNs and LPs. Fecal biomarkers of IBD such as calprotectin and lactoferrin increase significantly as infiltration of neutrophils in intestinal tracts [14]. Consistent with the previous studies, we showed that DSS induced increases in calprotectin and lactoferrin in rat feces, which were reduced by swimming. Thus, mitigation of neutrophil influx may be one reason that swimming protects against colon injury.

Consistent with the known anti-inflammatory and antioxidant effects of regular physical exercise, we found that regular swimming significantly reduced colon levels of TNF- $\alpha$, IL-1 $\beta$, IL- $6, \mathrm{KC}, \mathrm{NO}, \mathrm{PGE}_{2}$, and COX2 and serum levels of TNF- $\alpha$, IL- $1 \beta$, IL- $6, \mathrm{KC}$, and $\mathrm{PGE}_{2}$ in DSS-treated rats, which was accompanied by increased IL-10 production. TNF- $\alpha$, IL- $1 \beta$, IL- $6, \mathrm{NO}$, and $\mathrm{PGE}_{2}$ are key mediators of IBD $[18,19]$. Increased levels of IL10 are observed in the plasma of IBD patients and in the colon of rats with DSS-induced colitis; IL-10 attenuates the exaggerated inflammatory response $[20,21]$. It was reported that exercise increased IL-10 levels in DSSinduced colitis in adiponectin deficient mice and in TNBS-induced colitis in rats $[22,23]$. IL-10 elevation is thought to arise via a compensatory mechanism because this cytokine reduces mucosal inflammation [24, 25]. The detailed mechanism(s) by which swimming modulates the expression of these molecules requires further investigation. Recent studies indicate that regular exercise inhibits the activation of $\mathrm{NF}-\kappa \mathrm{B}$, which transcriptionally regulates COX2 and iNOS [26]. COX2 generates $\mathrm{PGE}_{2}$, which provokes intestinal hyperemia and edema. iNOS activation releases a surplus of $\mathrm{NO}$, which undermines colon integrity via the synthesis of peroxynitrite, a potent oxidizing agent formed during the reaction of NO with the superoxide anion [27]. In addition, TNF- $\alpha$ transcriptionally regulates iNOS and $\operatorname{COX} 2[28,29]$; swimming-mediated inhibition of TNF- $\alpha$ gene expression may also be a mechanistic link between such molecules and the suppression of colitis.

TNF- $\alpha$ is a pleiotropic cytokine that activates immune cells in IBD and subsequently promotes production of other pro-inflammatory cytokines [30]. IFN- $\gamma$ also plays a critical role in the induction and progression of colitis [31]. TNF- $\alpha$ - and IFN- $\gamma$-expressing $\mathrm{CD}^{+} \mathrm{T}$ cells, which are chemo-attracted to and activated by CCL2 and $\mathrm{KC}$, are highly involved in IBD exacerbation [32]. In this study, we showed that DSS-enhanced mucosal CCL2 and $\mathrm{KC}$ production, as well as the infiltration of TNF- $\alpha$ - and IFN- $\gamma$-expressing $\mathrm{CD}^{+} \mathrm{T}$ cells into the MLNs and LPs, was reduced when rats were treated with swimming exercise, suggesting that swimming reduces the levels of inflammatory mediators and mitigates infiltration of the injured site by activated $\mathrm{CD}^{+} \mathrm{T}$ cells.

Several clinical and experimental studies $[5,33]$ have implicated oxidative stress in IBD, in which the surge of ROS and NO generated by activated neutrophils causes intestinal injury. However, appropriate ROS production can be beneficial because specific adaptations are evoked. Such adaptations include increased antioxidant/oxidative damage-repairing enzyme activity and lower levels of oxidative stress [34]. Moreover, NF- $\kappa \mathrm{B}$ mediates a major oxidative stress-sensitive signal transduction pathway in mammalian tissues. Appropriate activation of the NF- $\kappa B$ signaling cascade increases expression of genes encoding important enzymes such as mitochondrial SOD, which maintain cellular oxidant/antioxidant homeostasis during exercise $[35,36]$. Nevertheless, excessive ROS production would cause overactivation of $\mathrm{NF}-\kappa \mathrm{B}$, resulting in disruption of the oxidant/antioxidant balance. In the present study, we demonstrated that swimming prevented intense oxidative stress and boosted antioxidant status in rats with DSS-induced colitis, as evidenced by reductions in ROS, MDA, and NO levels, iNOS activity, and NF-кB activation, in addition to the reinstatement of GSH and TAC levels and SOD and GPx activity. These findings agree with those of previous studies and reinforce the premise that swimming exerts antioxidant effects that protect against tissue damage [37]. It is postulated that swimming reduces the ROS to an appropriate level, and that mild oxidative stress stimulates the expression of certain antioxidant enzymes, which then maintain oxidant/ antioxidant homeostasis. However, these assumptions require further study.

Excessive exposure of intestinal mucosa to ROS under inflammatory conditions increases epithelial cell apoptosis and LDH release [15, 38], which likely alters epithelial barrier integrity and contributes to intestinal injury [7]. Apoptosis is partly regulated by the Bcl-2 family, which includes Bcl-2 and Bax. Bcl-2 is regarded as a pro-survival molecule, whereas Bax is a pro-apoptotic molecule as it binds to and antagonizes the effects of Bcl-2 [7]. An increased $\mathrm{Bax} / \mathrm{Bcl}-2$ ratio increases the release of AIF and cytochrome c from mitochondria into the cytosol, which then activates caspase-9 and caspase-3, ultimately causing apoptosis [39]. A previous study showed that exercise attenuates age-induced increases in the Bax/Bcl-2 ratio and reduces apoptosis [40]. The data presented herein revealed that swimming attenuated DSS-induced colon cell apoptosis by decreasing caspase- 3 activity, reducing AIF, cytochrome c, Bax, and Cl-caspase-3 protein levels, and simultaneously upregulating Bcl-2 expression, thereby ameliorating epithelial barrier dysfunction and improving colon architecture.

The present study has several limitations. First, we did not investigate whether the protective effects of swimming against DSS-induced chronic colitis are frequency- and/or intensity-dependent. Second, we did 
not undertake a detailed investigation of the potential signaling pathways involved in the process by which swimming protects against DSS-inducted chronic colitis. Other factors, which include aging, animal species, and treatment protocol, also need to be considered if we are planned to fully evaluate the protective effects of swimming.

In summary, we showed that swimming may be an adjunct or alternative therapy for DSS-induced colitis because it exerts anti-inflammatory, antioxidant, and antiapoptotic effects. The beneficial effects of swimming were associated with its ability to modulate the production of $\mathrm{NF}-\kappa \mathrm{B}, \mathrm{COX}-2$, and inflammatory mediators. Swimming mitigated oxidative stress and boosted enzymatic/nonenzymatic antioxidant defenses. Moreover, swimming reduced caspase-3 activity and pro-apoptotic gene expression, with concomitant upregulation of Bcl-2. These findings suggest that swimming is an effective therapy for DSS-induced chronic colitis. However, the exact molecular mechanisms and signaling networks underlying the positive effects of swimming still need to be examined. Thus, further studies should investigate the potential therapeutic efficacy of swimming for the management of IBD following the manifestation of symptoms.

\section{MATERIALS AND METHODS}

\section{Materials}

All reagents were obtained from Sigma (St. Louis, MO, USA) unless otherwise noted. All chemicals were of the highest commercial grade available. Rabbit anti-

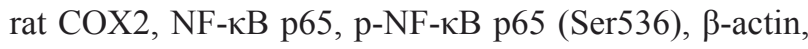
and lamin $\mathrm{B}$ antibodies were purchased from Abcam (Cambridge, UK). Rabbit anti-rat AIF, Bax, Bcl-2, caspase-3, and Cl-caspase-3 were acquired from Abnova (Taiwan, China). Horseradish peroxidase-conjugated anti-rabbit IgG was procured from Chemicon (Temecula, CA, USA). 2',7'-Dichlorodihydrofluorescein diacetate, acetyl ester, and dihydroethidium were purchased from Molecular Probes (Eugene, OR, USA). DSS (MW = 36,000 to 50,000; ICN Biomedicals Inc., Aurora, Ohio, USA) was dissolved in regular drinking water. All suspensions were freshly prepared before use.

\section{Animals and ethics statement}

Male Sprague-Dawley rats (6 weeks old and weighing $140 \mathrm{~g}$ to $160 \mathrm{~g}$ ) were obtained from the Laboratory Animal Center of the Fourth Military Medical University and maintained at a comfortable temperature $\left(22^{\circ} \mathrm{C}\right.$ to $\left.24^{\circ} \mathrm{C}\right)$ and relative humidity of $40 \%$ to $70 \%$, with a regular $12 \mathrm{~h}$ day/night cycle, and access to standard laboratory chow and tap water ad libitum. All experimental protocols were approved by the Institutional Animal Research Ethics board of the 150th Central Hospital of PLA. Each animal received humane treatment in full compliance with the National Institutes of Health (Bethesda, MD, USA) criteria for the Care and Use of Laboratory Animals. Rats were sacrificed under anesthesia by intraperitoneal injection of sodium pentobarbital, and all efforts to minimize suffering were made. Euthanasia by sodium pentobarbital was performed in accordance with the American Veterinary Medical Association Guidelines on Euthanasia, June 2007.

\section{DSS-induced chronic colitis and weight-unloaded swimming exercise}

After transport, rats were acclimatized for $7 \mathrm{~d}$. A DSS-induced chronic colitis model was established as previously described [41]. The protocol involved three 5 $\mathrm{d}$ cycles of $2 \%$ DSS separated by $14 \mathrm{~d}$ intervals. Animals without DSS treatment were used as a vehicle control and had access to regular drinking water during the study.

Weight-unloaded swimming training was performed as previously described [37], with slight modifications. Swimming took place in a $120 \mathrm{~cm}$ glass container filled with water to a depth of $80 \mathrm{~cm}$ and maintained at $35^{\circ} \mathrm{C}$ to $36^{\circ} \mathrm{C}$. Rats were acclimatized to the training pool for 1 week prior to swimming training. Acclimation entailed swimming for gradually increasing times $(30,40,50,60$, $70,80$, and $90 \mathrm{~min} / \mathrm{d})$. After acclimation, rats were trained for 7 weeks (either $1 \mathrm{~h} / \mathrm{d}$ or $1.5 \mathrm{~h} / \mathrm{d}$ on $5 \mathrm{~d} /$ week). DSS administration began on the first swimming day. Untrained rats were kept in the same glass containers, which contained water (maintained at $35^{\circ} \mathrm{C}$ ) at a depth of $4 \mathrm{~cm}$.

\section{Sample collection and preparation}

After 7 weeks of swimming exercise, rats were fasted for $4 \mathrm{~h}$. Samples of blood was centrifuged for 15 $\min$ at $1,000 \times g$, and serum samples were harvested and stored at $-20^{\circ} \mathrm{C}$ for detection of $\mathrm{LDH}$ release and inflammatory mediators. At the time of euthanasia, feces were collected and stool samples were extracted in the extraction buffer (Sigma), centrifuged for 5 min at 13,000 $\times g$, and then used for the measurement of calprotectin and lactoferrin. After all animals were euthanized, laparotomy was performed immediately. The spleen was dissected for photoing and weighing. The colon was removed from the distal end of the cecum to the rectum, freed of adherent adipose tissue, split longitudinally, and washed with icecold saline to remove fecal residue and photographed. Isolated colons were utilized for histopathological, immunohistochemical, biochemical investigation, and other analyses. 


\section{Experimental design}

Ninety rats were randomly divided into six groups ( $n=15$ /group) as follows: (1) Vehicle + Sedentary group $(\mathrm{Ctrl}+\mathrm{Sed})[$ rats were administered regular drinking water without swimming exercise]; (2) Vehicle + Exercise group $1(\mathrm{Ctrl}+\mathrm{Ex} 1)$ [identical to the Ctrl + Sed group, except that rats swam for $1 \mathrm{~h} / \mathrm{d}$ ]; (3) Vehicle + Exercise group 2 $(\mathrm{Ctrl}+\mathrm{Ex} 2)$ [identical to the Ctrl + Sed group, except that rats swam for $1.5 \mathrm{~h} / \mathrm{d}$ ]; (4) DSS + Sedentary group (DSS + Sed) [identical to the Ctrl + Sed group, except that rats received $2 \% \mathrm{DSS} /$ water instead of regular drinking water]; (5) DSS + Exercise group 1 (DSS + Ex 1) [identical to DSS + Sed group, except that rats swam for $1 \mathrm{~h} / \mathrm{d}]$; and (6) DSS + Exercise group 2 (DSS + Ex 2) [identical to DSS + Sed group, except that rats swam for $1.5 \mathrm{~h} / \mathrm{d}$ ].

\section{Measurement of BW, spleen weight and colon length}

BW was measured before and after adaptation, and once a week throughout the experimental period. After 7 weeks of swimming exercise, rats were fasted for $4 \mathrm{~h}$ and then euthanized with sodium pentobarbital. The spleen and entire colon were removed, and spleen weight and colon length were measured.

\section{Determination of colon barrier function}

Intestinal barrier function was examined as previously described [41]. In brief, colon barrier integrity was assessed according to its permeability to FITCdextran (MW $=4,000$; FD4). After $4 \mathrm{~h}$ of fasting, rats received FD4 by gavage [600 $\mathrm{mg} / \mathrm{kg} \mathrm{BW}$ in $0.2 \mathrm{~mL}$ of sodium-phosphate buffered saline (PBS)]. Plasma samples were obtained $5 \mathrm{~h}$ after the procedure, and fluorescence was measured.

\section{Histological examination and quantitation of inflammation}

Colon tissues were fixed overnight in PBSbuffered $10 \%$ formalin, embedded in paraffin, nonserially sectioned ( $4 \mu \mathrm{m}$ thick), and mounted on poly-Llysine-covered slides. After deparaffinization in xylene and rehydration in a graded series of ethanol solutions, the sections were stained with hematoxylin and eosin (HE). Histological changes were evaluated by two independent pathologists as previously described [42]; neither pathologist had any knowledge of the treatment regimen received by each animal. Inflammation was graded according to its extent (focal, multifocal, diffuse, or extensive) and depth/penetration into the LP, submucosa, muscularispropria, and sub-serosa. The degree of inflammation was scored from 0 to 4 , where 0 indicates no observable inflammation and 4 denotes severe inflammation and/or ulceration/erosion. Tissue histology was recorded upon observation under a light microscope (Leica, Wetzlar, Germany) equipped with image capturing software. Image-Pro Discovery software (Media Cybernetics Inc., Silver Spring, MD, USA) was used to measure crypt depth, which was determined from 10 to 15 independent measurements on at least three different tissue sections per rat.

\section{IHC and staining evaluation}

After deparaffinization and rehydration, colon sections were submerged in citrate buffer ( $\mathrm{pH}$ 6.0) and boiled in an autoclave at $121^{\circ} \mathrm{C}$ for $3 \mathrm{~min}$ for antigen retrieval. The slides were then cooled to room temperature. Endogenous peroxidase was quenched with $0.3 \% \mathrm{H}_{2} \mathrm{O}_{2}$ in methanol for $15 \mathrm{~min}$. Nonspecific adsorption was minimized by incubating the section in $10 \%$ normal goat serum (Gibco, BRL, UK) in PBS for $20 \mathrm{~min}$. Sections were incubated overnight with primary antibodies and then with a biotinylated secondary antibody (ChemMateEnVision Kit; DAKO, Hamburg, Germany) for $15 \mathrm{~min}$. The reaction products were visualized using diaminobenzidine (DAB) substrate (Maixin Biotech., Fuzhou, China). Sections were counterstained with commercial hematoxylin (Maixin Biotech.), dehydrated, and examined under a light microscope (Leica).

The NF- $\mathrm{KB}$ p65 and COX2 protein levels were evaluated by a blind way in 10 fields under $100 \times$ magnification from each slide. IHC staining was scored as previously described [43]. One hundred cells per field were categorized as follows: " 0 ", $0 \%$, no staining; 1 to $25 \%$ of cells were stained; " 2 ", 26 to $50 \%$ of cells were stained; and " 3 ", $>50 \%$ of cells were stained.

\section{Measurement of MPO activity}

MPO activity, an indicator of neutrophil infiltration into the colon tissue, was measured using commercial kits from Molecular Probes (Eugene, OR, USA), as previously described [44]. Briefly, approximately $1 \mathrm{~g}$ of wet colon tissue was homogenized in $0.5 \%$ hexadecyltrimethylammonium bromide dissolved in PBS. The tissue homogenate was centrifuged at 12,500 $\times g$ for 40 min at $4^{\circ} \mathrm{C}$, and the supernatant was collected to detect MPO activity, which was defined by a change in the absorbance measured by a spectrophotometer (DU 640B; Beckman) at $590 \mathrm{~nm}$. Results were expressed as absorbance units per gram of wet tissue. 


\section{Flow cytometry analysis}

Cells were freshly isolated from MLN and LP, as previously described [16]. Fc receptors on the cells were pre-blocked at $4^{\circ} \mathrm{C}$ for $15 \mathrm{~min}$. The cells were washed with fluorescence-activated cell sorting (FACS) staining buffer (PBS containing 1\% bovine serum albumin) and stained with Cy-conjugated anti-CD3 (145-2C11) and FITC-conjugated Ly6G (neutrophils; BD Pharmingen) antibodies at $4^{\circ} \mathrm{C}$ for $30 \mathrm{~min}$, with occasional shaking. The cells were washed twice with FACS staining buffer, resuspended in BD Cytofix/Cytoperm (BD Pharmingen) solution for $20 \mathrm{~min}$, and then washed twice in $\mathrm{BD}$ Perm/Wash solution. To examine intracellular cytokine expression, the resuspended, fixed, and permeabilized cells were stained with predetermined allophycocyanin fluorochrome-conjugated anti-cytokine antibodies (antiTNF- $\alpha$ and anti-IFN- $\gamma$ ) at $4^{\circ} \mathrm{C}$ for $30 \mathrm{~min}$ in the dark. Lymphocytes were thoroughly washed with FACS staining buffer and analyzed by flow cytometry (BD Aria $\Pi$, Becton Dickinson, USA).

\section{Measurement of serum LDH}

The blood samples collected from all animals were centrifuged, serum separated and used to determine LDH activity using BioVision kit (Milpitas, CA, USA).

\section{ELISAs}

The entire colon was removed from the distal end of the cecum to the rectum, cut longitudinally, thoroughly rinsed in sterile $20 \mathrm{mM}$ PBS ( $\mathrm{pH} 7.4$ ), spread onto the bottoms of six-well dishes, and incubated overnight in $1 \mathrm{~mL}$ of culture medium. The supernatant levels of TNF- $\alpha$, IL-1 $\beta$, IL-6, IL-10, KC, CCL2, and PGE 2 and serum levels of TNF- $\alpha$, IL-1 $\beta$, IL- $6, \mathrm{KC}$, and $\mathrm{PGE}_{2}$ were measured using commercial ELISA kits (R\&D Systems, Minneapolis, MN, USA). Fecal levels of calprotectin and lactoferrin were determined using ELISA kits (Abbexa, Cambridge, UK). Optical density (OD) was measured on an ELISA plate scanner (CA94089, Molecular Devices, Sunnyvale, Canada).

\section{Measurement of ROS production}

ROS levels were determined by measuring the oxidative conversion of DCFH-DA to the fluorescent compound DCF. Briefly, colon tissues were homogenized in PBS (pH 7.4) and centrifuged at $10,000 \times g$ for $20 \mathrm{~min}$ at $4^{\circ} \mathrm{C}$, and the supernatant was collected, washed with warm HBSS, and incubated in HBSS containing $10 \mu \mathrm{M}$ DCFH-DA at $37^{\circ} \mathrm{C}$ for $30 \mathrm{~min}$. DCF fluorescence was determined at $485 \mathrm{~nm}$ (excitation) and $520 \mathrm{~nm}$ (emission) in a fluorescence microplate reader (Safire2, Tecan, Switzerland).

\section{Measurement of MDA}

MDA levels were determined using the thiobarbituric acid assay as reported by Buege and Aust [45]. Absorbance was recorded at $535 \mathrm{~nm}$, and the results were expressed as $\mathrm{nmol} / \mathrm{g}$ tissue.

\section{Measurement of iNOS activity}

iNOS activity was measured by monitoring the conversion of arginine to citrullinein, as previously described [46]. Briefly, an aliquot of colon tissue lysate was incubated with L- $\left[{ }^{3} \mathrm{H}\right]$ arginine along with essential substrates and cofactors (tetrahydrobiopterin, nicotinamide adenine dinucleotide phosphate, and flavin adenine dinucleotide). The amount of product, namely, L- $\left[{ }^{3} \mathrm{H}\right]$ citrulline, was calculated after liquid scintillation counting. To quantify iNOS activity, ethylene diamine tetraacetic acid (EDTA) and ethylene glycol tetraacetic acid (EGTA) were added sequentially to the incubation buffer. An appropriate blank, including $1 \mathrm{mM} \mathrm{L-NAME,}$ a competitive iNOS inhibitor, was used to exclude the effects of nonspecific L-arginine metabolism. The iNOS activity in the citrulline assay was analyzed according to the degree of L-NAME inhibition in the EDTA-EGTA sample, and its expression was measured in Units (1 Unit $=1 \mathrm{pM}$ L-citrulline $/ \mathrm{mg}$ protein $/ \mathrm{min}$ ).

\section{Measurement of nitrite}

The production of nitrite, an indicator of NO synthesis, was measured in a colorimetric reaction with the Griess reagent [47]. Briefly, colon tissues were homogenized in PBS (pH 7.4) and centrifuged at 10,000 $\times g$ at $4^{\circ} \mathrm{C}$ for $20 \mathrm{~min}$. The supernatant was collected and mixed with equal $(1: 1)$ volumes of Griess reagent $[0.1 \%$ $\mathrm{N}-(1$-naphthyl) ethylenediaminedihydrochloride, 1\% sulfanilamide, and $\left.2.5 \% \mathrm{H}_{3} \mathrm{PO}_{4}\right]$. A 96-well microplate reader (Spectra MAX 340PC; Molecular Devices) was used to measure the absorbance at $540 \mathrm{~nm}$. Data were analyzed using Softmax Pro software. Sodium nitrite, dissolved in double-distilled water, was used as a standard.

\section{Measurement of GSH}

GSH levels in the supernatants of colon homogenates were determined using 5,5'-dithiobis 2-nitrobenzoic acid (DTNB) reagent as previously described [48]. The OD of the colored product was read at $412 \mathrm{~nm}$, and the results were expressed as $\mathrm{nmol} / \mathrm{g}$ tissue. 


\section{Determination of TAC}

TAC was determined using the Cayman total antioxidant assay kit, according to the manufacturer's instructions. The assay depends on the ability of antioxidants in the supernatants of colon homogenates to inhibit the metmyoglobin-mediated oxidation of ABTS [2,2-azino-di-(3-ethylbenzthiazoline sulfonate)]. The amount of oxidized product was estimated by measuring the absorbance at $405 \mathrm{~nm}$. The antioxidant capacity of the sample was compared with that of Trolox, a water-soluble tocopherol analogue, and the results were expressed as mmol of Trolox equivalent/g tissue.

\section{Measurement of SOD activity}

SOD activity was determined by the ability of colonic SOD to inhibit the auto-oxidation of pyrogallol, as previously described [49]. The change in absorbance at $420 \mathrm{~nm}$ was measured at $1 \mathrm{~min}$ intervals for $3 \mathrm{~min}$. One unit of SOD is defined as the amount of enzyme that affords $50 \%$ inhibition of pyrogallol auto-oxidation in 1 $\mathrm{min}$. The results were expressed as $\mathrm{U} / \mathrm{mg}$ protein.

\section{Determination of GPx activity}

GPx activity was determined according to the method of Paglia and Valentine [50]. This method depends on the ability of the enzyme to oxidize GSH, which was monitored by recording the decrease in absorbance of NADPH at $340 \mathrm{~nm}$. One unit of enzyme is defined as the amount of enzyme that oxidizes $1 \mathrm{mmol} \mathrm{NADPH} / \mathrm{min}$ at $25^{\circ} \mathrm{C}$.

\section{Caspase- 3 activity assay}

Caspase-3 activity was detected using the Caspase-3/CPP32 Colorimetric Assay Kit (BioVision), according to the manufacturer's instructions. An aliquot of homogenate supernatant was incubated with the labeled substrate, DEVD-pNA (acetyl-Asp-Glu-Val-Asp p-nitroanilide). Cleavage of the peptide by the caspase releases the chromophore, pNA. Results were read at $405 \mathrm{~nm}$ in a microplate reader (Bio-Tek instruments Inc., Winooski, VT, USA) and expressed as the fold change in caspase-3 activity.

\section{Western blot analysis}

Colon tissue samples were homogenized in tissue lysis and extraction buffer containing protease inhibitor cocktail set III (EMD Chemicals Inc., Germany). Crude homogenates were repetitively filtered and centrifuged at $15,000 \times g$ at $4{ }^{\circ} \mathrm{C}$ for $25 \mathrm{~min}$. The supernatants were collected as cytosolic fractions. The nuclei-rich pellets were resuspended in nuclear protein extraction buffer containing $20 \mathrm{mM}$ 4-(2-hydroxyethyl)-1piperazineethanesulfonic acid (pH 7.9), 10\% glycerol, $1 \mathrm{mM}$ dithiothreitol, $400 \mathrm{mM} \mathrm{NaCl}, 1 \mathrm{mM}$ EDTA, and cocktail set III. After $20 \mathrm{~min}$ of centrifugation at 15,000 $\times$ $g$ at $4^{\circ} \mathrm{C}$, the supernatants containing nuclear proteins were pooled. NF- $\mathrm{KB}$ p65 levels in the nuclear fractions and the levels of all other proteins in the cytosolic fractions were quantified. The two extracts (cytosolic and nuclear) were boiled, and the proteins were separated by sodium dodecyl sulfate polyacrylamide gel electrophoresis, electrotransferred onto nitrocellulose membranes, and immunoblotted with appropriate primary antibodies. Equivalent sample loading was confirmed by probing with rabbit monoclonal antibodies against rat $\beta$-actin and lamin B. Bands were detected using an enhanced Chemiluminescence Assay Kit (Pierce, Rockford, IL, USA).

\section{Statistical analysis}

All data were expressed as the mean \pm standard derivation (SD). Intergroup differences were analyzed using Student's $t$-test (two-tailed, unpaired) or one-way ANOVA, followed by Dunnett's post-hoc test where appropriate. GraphPad statistical software (GraphPad Software, Inc., San Diego, CA, USA) was used for data analysis. $P<0.05$ was considered to be statistically significant.

\section{Abbreviations}

AIF, apoptosis-inducing factor; BW, body weight; Cl-caspase-3, cleaved-caspase-3; COX2, cyclooxygenase 2; DAB, diaminobenzidine; DSS, dextran sulfate sodium; ELISA, enzyme-linked immunosorbent assay; FACS, fluorescence-activated cell sorting; FD4, FITC-dextran; GPx, glutathione peroxidase; GSH, glutathione; HE, hematoxylin and eosin; IBD, inflammatory bowel disease; IFN- $\gamma$, interferon- $\gamma$; IHC, Immunohistochemistry; IL, interleukin; iNOS, inducible nitric oxide synthase; LP, lamina propria; MDA, malondialdehyde; MLN, mesenteric lymph nodes; MPO, myeloperoxidase; NF$\kappa \mathrm{B}$, nuclear factor- $\kappa \mathrm{B}$; NO, nitric oxide; PBS; sodiumphosphate buffer; $\mathrm{PGE}_{2}$, prostaglandin $\mathrm{E}_{2}$; ROS, reactive oxygen species; SOD, superoxide dismutase; TAC, total antioxidant capacity; TNF- $\alpha$, tumor necrosis factor- $\alpha$; UC, ulcerative colitis.

\section{ACKNOWLEDGMENTS}

This research was supported by the National Key Research Program of China (No. 2014ZX09J14101-01A). 


\section{CONFLICTS OF INTEREST}

The authors have no conflicts of interest to declare.

\section{REFERENCES}

1. Axelrad JE, Lichtiger S and Yajnik V. Inflammatory bowel disease and cancer: The role of inflammation, immunosuppression, and cancer treatment. World J Gastroenterol. 2016; 22(20):4794-4801.

2. Randhawa PK, Singh K, Singh N and Jaggi AS. A review on chemical-induced inflammatory bowel disease models in rodents. The Korean journal of physiology \& pharmacology. 2014; 18(4):279-288.

3. Hanauer SB. Inflammatory bowel disease: epidemiology, pathogenesis, and therapeutic opportunities. Inflamm Bowel Dis. 2006; 12 Suppl 1:S3-9.

4. Fengming $\mathrm{Y}$ and Jianbing W. Biomarkers of inflammatory bowel disease. Disease markers. 2014; 2014:710915.

5. Zhu H and Li YR. Oxidative stress and redox signaling mechanisms of inflammatory bowel disease: updated experimental and clinical evidence. Exp Biol Med (Maywood). 2012; 237(5):474-480.

6. Kretzmann NA, Fillmann H, Mauriz JL, Marroni CA, Marroni N, Gonzalez-Gallego J and Tunon MJ. Effects of glutamine on proinflammatory gene expression and activation of nuclear factor kappa B and signal transducers and activators of transcription in TNBS-induced colitis. Inflamm Bowel Dis. 2008; 14(11):1504-1513.

7. Becker C, Watson AJ and Neurath MF. Complex roles of caspases in the pathogenesis of inflammatory bowel disease. Gastroenterology. 2013; 144(2):283-293.

8. Astrom MB, Feigh M and Pedersen BK. Persistent lowgrade inflammation and regular exercise. Front Biosci (Schol Ed). 2010; 2:96-105.

9. Cook MD, Martin SA, Williams C, Whitlock K, Wallig MA, Pence BD and Woods JA. Forced treadmill exercise training exacerbates inflammation and causes mortality while voluntary wheel training is protective in a mouse model of colitis. Brain Behav Immun. 2013; 33:46-56.

10. Avula CP, Muthukumar AR, Zaman K, McCarter R and Fernandes $\mathrm{G}$. Inhibitory effects of voluntary wheel exercise on apoptosis in splenic lymphocyte subsets of C57BL/6 mice. J Appl Physiol (1985). 2001; 91(6):2546-2552.

11. Meredith-Jones $\mathrm{K}$, Waters $\mathrm{D}$, Legge $\mathrm{M}$ and Jones L. Upright water-based exercise to improve cardiovascular and metabolic health: a qualitative review. Complement Ther Med. 2011; 19(2):93-103.

12. Demarzo MM, Martins LV, Fernandes CR, Herrero FA, Perez SE, Turatti A and Garcia SB. Exercise reduces inflammation and cell proliferation in rat colon carcinogenesis. Med Sci Sports Exerc. 2008; 40(4):618621.

13. Natsui M, Kawasaki K, Takizawa H, Hayashi SI, Matsuda
Y, Sugimura K, Seki K, Narisawa R, Sendo F and Asakura $\mathrm{H}$. Selective depletion of neutrophils by a monoclonal antibody, RP-3, suppresses dextran sulphate sodiuminduced colitis in rats. J Gastroenterol Hepatol. 1997; 12(12):801-808.

14. Sipponen T. Diagnostics and prognostics of inflammatory bowel disease with fecal neutrophil-derived biomarkers calprotectin and lactoferrin. Digestive diseases (Basel, Switzerland). 2013; 31(3-4):336-344.

15. Kruidenier L, Kuiper I, Lamers CB and Verspaget HW. Intestinal oxidative damage in inflammatory bowel disease: semi-quantification, localization, and association with mucosal antioxidants. J Pathol. 2003; 201(1):28-36.

16. Cui X, Jin Y, Hofseth AB, Pena E, Habiger J, Chumanevich A, Poudyal D, Nagarkatti M, Nagarkatti PS, Singh UP and Hofseth LJ. Resveratrol suppresses colitis and colon cancer associated with colitis. Cancer Prev Res (Phila). 2010; 3(4):549-559.

17. Morohoshi Y, Matsuoka K, Chinen H, Kamada N, Sato T, Hisamatsu T, Okamoto S, Inoue N, Takaishi H, Ogata $\mathrm{H}$, Iwao $\mathrm{Y}$ and Hibi T. Inhibition of neutrophil elastase prevents the development of murine dextran sulfate sodiuminduced colitis. J Gastroenterol. 2006; 41(4):318-324.

18. Strober W, Zhang F, Kitani A, Fuss I and Fichtner-Feigl S. Proinflammatory cytokines underlying the inflammation of Crohn's disease. Current opinion in gastroenterology. 2010; 26(4):310-317.

19. Strober $\mathrm{W}$ and Fuss IJ. Proinflammatory cytokines in the pathogenesis of inflammatory bowel diseases. Gastroenterology. 2011; 140(6):1756-1767.

20. Shah N, Kammermeier J, Elawad M and Glocker EO. Interleukin-10 and interleukin-10-receptor defects in inflammatory bowel disease. Current allergy and asthma reports. 2012; 12(5):373-379.

21. Kole A and Maloy KJ. Control of intestinal inflammation by interleukin-10. Current topics in microbiology and immunology. 2014; 380:19-38.

22. Saxena A, Fletcher E, Larsen B, Baliga MS, Durstine JL and Fayad R. Effect of exercise on chemically-induced colitis in adiponectin deficient mice. Journal of inflammation (London, England). 2012; 9(1):30.

23. Szalai Z, Szasz A, Nagy I, Puskas LG and Kupai K. Antiinflammatory effect of recreational exercise in TNBSinduced colitis in rats: role of NOS/HO/MPO system. 2014; 2014:925981.

24. Gasche C, Bakos S, Dejaco C, Tillinger W, Zakeri S and Reinisch W. IL-10 secretion and sensitivity in normal human intestine and inflammatory bowel disease. Journal of clinical immunology. 2000; 20(5):362-370.

25. Li MC and He SH. IL-10 and its related cytokines for treatment of inflammatory bowel disease. World $\mathrm{J}$ Gastroenterol. 2004; 10(5):620-625.

26. Greten FR, Eckmann L, Greten TF, Park JM, Li ZW, Egan LJ, Kagnoff MF and Karin M. IKKbeta links inflammation 
and tumorigenesis in a mouse model of colitis-associated cancer. Cell. 2004; 118(3):285-296.

27. Soufli I, Toumi R, Rafa H and Touil-Boukoffa C. Overview of cytokines and nitric oxide involvement in immunopathogenesis of inflammatory bowel diseases. World journal of gastrointestinal pharmacology and therapeutics. 2016; 7(3):353-360.

28. Kleinert H, Pautz A, Linker K and Schwarz PM. Regulation of the expression of inducible nitric oxide synthase. European journal of pharmacology. 2004; 500(1-3):255266.

29. Qiu J, Yuan H, Chen S, Zhou Y, Song D and Chen R. TNFalpha up-regulates COX-2 in chronic progressive nephropathy through nuclear accumulation of RelB and NFkappaB2. Archives of physiology and biochemistry. 2016; 122(2):88-93.

30. Olesen CM, Coskun M, Peyrin-Biroulet L and Nielsen $\mathrm{OH}$. Mechanisms behind efficacy of tumor necrosis factor inhibitors in inflammatory bowel diseases. Pharmacology \& therapeutics. 2016; 159:110-119.

31. Xu XR, Liu CQ, Feng BS and Liu ZJ. Dysregulation of mucosal immune response in pathogenesis of inflammatory bowel disease. World J Gastroenterol. 2014; 20(12):32553264.

32. Olsen T, Goll R, Cui G, Husebekk A, Vonen B, Birketvedt GS and Florholmen J. Tissue levels of tumor necrosis factor-alpha correlates with grade of inflammation in untreated ulcerative colitis. Scand J Gastroenterol. 2007; 42(11):1312-1320.

33. Balmus IM, Ciobica A, Trifan A and Stanciu C. The implications of oxidative stress and antioxidant therapies in Inflammatory Bowel Disease: Clinical aspects and animal models. Saudi journal of gastroenterology. 2016; 22(1):317.

34. Schieber M and Chandel NS. ROS function in redox signaling and oxidative stress. Current biology. 2014; 24(10):R453-462.

35. Ji LL, Gomez-Cabrera MC and Vina J. Exercise and hormesis: activation of cellular antioxidant signaling pathway. Ann N Y Acad Sci. 2006; 1067:425-435.

36. Kramer HF and Goodyear LJ. Exercise, MAPK, and NFkappaB signaling in skeletal muscle. J Appl Physiol (1985). 2007; 103(1):388-395.

37. Venditti P and Di Meo S. Antioxidants, tissue damage, and endurance in trained and untrained young male rats. Arch Biochem Biophys. 1996; 331(1):63-68.

38. Crespo I, San-Miguel B, Prause C, Marroni N, Cuevas MJ, Gonzalez-Gallego J and Tunon MJ. Glutamine treatment attenuates endoplasmic reticulum stress and apoptosis in TNBS-induced colitis. PLoS One. 2012; 7(11):e50407.

39. Redza-Dutordoir M and Averill-Bates DA. Activation of apoptosis signalling pathways by reactive oxygen species. Biochimica et biophysica acta. 2016; 1863(12):2977-2992.
40. Kwak HB, Song W and Lawler JM. Exercise training attenuates age-induced elevation in $\mathrm{Bax} / \mathrm{Bcl}-2$ ratio, apoptosis, and remodeling in the rat heart. FASEB J. 2006; 20(6):791-793.

41. Cooks T, Pateras IS, Tarcic O, Solomon H, Schetter AJ, Wilder S, Lozano G, Pikarsky E, Forshew T, Rosenfeld N, Harpaz N, Itzkowitz S, Harris CC, Rotter V, Gorgoulis VG and Oren M. Mutant p53 prolongs NF-kappaB activation and promotes chronic inflammation and inflammationassociated colorectal cancer. Cancer Cell. 2013; 23(5):634646.

42. Jin Y, Kotakadi VS, Ying L, Hofseth AB, Cui X, Wood PA, Windust A, Matesic LE, Pena EA, Chiuzan C, Singh NP, Nagarkatti M, Nagarkatti PS, Wargovich MJ and Hofseth LJ. American ginseng suppresses inflammation and DNA damage associated with mouse colitis. Carcinogenesis. 2008; 29(12):2351-2359.

43. Chen M, Gao L and Chen P. Serotonin-Exacerbated DSSInduced Colitis Is Associated with Increase in MMP3 and MMP-9 Expression in the Mouse Colon. 2016; 2016:5359768.

44. Balaha M, Kandeel S and Elwan W. Garlic oil inhibits dextran sodium sulfate-induced ulcerative colitis in rats. Life sciences. 2016; 146:40-51.

45. Buege JA and Aust SD. Microsomal lipid peroxidation. Methods Enzymol. 1978; 52:302-310.

46. Barrientos A, Kenyon L and Moraes CT. Human xenomitochondrial cybrids. Cellular models of mitochondrial complex I deficiency. J Biol Chem. 1998; 273(23):14210-14217.

47. Zhang H, Deng A, Zhang Z, Yu Z, Liu Y, Peng S, Wu L, Qin $H$ and Wang W. The protective effect of epicatechin on experimental ulcerative colitis in mice is mediated by increasing antioxidation and by the inhibition of NF-kappaB pathway. Pharmacological reports. 2016; 68(3):514-520.

48. Mokhber Maleki E, Eimani H, Bigdeli MR, Golkar Narenji A and Abedi R. Effects of Crocin Supplementation during In Vitro Maturation of Mouse Oocytes on Glutathione Synthesis and Cytoplasmic Maturation. International journal of fertility \& sterility. 2016; 10(1):53-61.

49. Xu C, Liu S, Liu Z, Song F and Liu S. Superoxide generated by pyrogallol reduces highly water-soluble tetrazolium salt to produce a soluble formazan: a simple assay for measuring superoxide anion radical scavenging activities of biological and abiological samples. Analytica chimica acta. 2013; 793:53-60.

50. Paglia DE and Valentine WN. Studies on the quantitative and qualitative characterization of erythrocyte glutathione peroxidase. J Lab Clin Med. 1967; 70(1):158-169. 